\title{
Theme: Pediatric Allied Specialties
}

\begin{abstract}
Association between congenital nasolacrimal duct obstruction and mode of delivery at birth. (JAAPOS. 2018;22:381-85).
\end{abstract}

Congenital nasolacrimal duct obstruction is one of the commonest ophthalmological problems encountered in newborns.This study, conducted with an objective to find out any association of mode of delivery with the incidence and treatment outcome of nasolacrimal duct obstruction, showed a significantly higher risk (OR 1.55) of nasolacrimal duct obstruction in term babies born through caesarean section. Absence of spontaneous resolution and failure to respond to first line probing was also much more common in babies delivered via caesarean section. With rising rates of caesarean sections, pediatricians may need to screen all newborns for congenital nasolacrimal duct obstruction at birth and subsequent visits.

Femoral neck aspiration for the diagnosis of osteomyelitis in children with septic hip ( $\mathrm{J}$ Pediatr Orthop. 2018;38:532-36).

This study was conducted to determine whether femoral aspiration conducted concomitantly with irrigation and debridement of the septic hip aids in microorganism and osteomyelitis identification and alters the treatment plan. The study also compared preoperative magnetic resonance imaging (MRI) with femoral aspiration for diagnosis of osteomyelitis occurring along with septic arthritis. The authors concluded that though femoral aspiration did not improve microorganism identification, the benefits far outweighed the risks as a modality for diagnosis when compared to MRI (sensitivity/specificity for femoral aspiration vs MRI was 100\%/ $100 \%$ vs $38 \% / 95 \%$ ). No major side effects were noted after femoral aspiration whereas missed or delayed diagnosis led to significant morbidity.

Sensorineural hearing loss in children with Down syndrome (Int J Pediatr Otorhinolaryngol. 2019;116:16872).

Hearing loss is the most common otorhinolaryngological complaint associated with Down Syndrome. Conductive deafness is the most common type of deafness in Down syndrome. The prevalence and underlying etiology of sensorineural hearing loss (SNHL) has not been well-defined in children with Down syndrome. The present study was conducted to evaluate prevalence, severity, laterality and underlying etiology of SNHL in a cohort of children with Down syndrome. They found that SNHL was present in only $4.5 \%$ of children with hearing loss, of which $40 \%$ was congenital in origin. Mild, moderate and profound hearing loss each was observed in one-third of the patients. Though etiological diagnosis was not evaluated in all cases with SNHL, cochlear nerve deficiency was the most common cause found in those assessed. As working parents are moving towards late pregnancies, the incidence of Down syndrome has also increased. Hearing loss occurring in nearly two-third of these children, if evaluated in time, can significantly help in improving cognition and speech.

\section{Abametapir for the treatment of head lice (Pediatr (f Dermatol. 2018;35:616-21).}

Synthetic pyretheroids (Permethrin) have been the most widely used drugs for treatment of head lice. Lately, there have been reports of progressively increasing resistance to this drug. Newer drugs have targeted metalloproteinase, considered to be essential for louse egg hatching. Abametapir, a metalloproteinase inhibitor, is one such drug. The present study was conducted to assess the efficacy of abametapir lotion $(0.74 \%)$ to clear head louse infestation after a single application. The study concluded that the drug was effective at clearing active head louse infestations through day-14 in participants aged 6 months and older $(P<0.001)$; though, some transient side effects were noted.

\section{丹}

Early clean intermittent catheterization versus expectant management in children with spina bifida (https://doi.org/10.1016/j.jpedsurg.2018.10.096).

Renal damage and renal scarring following bladder involvement is well documented in spina bifida. The damage starts even earlier than 6 months of age. Early clean catheterization has well been into practice since late nineties. The above study was planned to assess if early clean intermittent catheterization carried for all newborn infants with spina bifida over 13 year period was associated with a reduction in scar rate determined by DMSA as compared to previous group managed expectantly. Case records and DMSA reports were studied retrospectively, and authors concluded that renal scarring rates were much lower in the recent cohort managed with early clean intermittent catheterization when compared to previous group $(P<0.002)$. Also, the age of onset of renal scarring was later in the recent cohort.

Increased risk for neurodevelopmental disorders in children with orofacial clefts $(\mathrm{J} \mathrm{Am} \mathrm{Acad} \mathrm{Child} \mathrm{Adolesc}$ Psych. 2018;57:876-83).

Orofacial cleft deformity is one of the common congenital malformations. This condition requires a multidisciplinary approach, and regular assessments of facial growth and speech. In addition to these, children also needs to be assessed for cognitive and language skills. The above study was done to investigate the risk of psychiatric diagnoses in individuals with non-syndromic orofacial clefts. It was seen that these children have a significantly higher risk of any psychiatric disorder, intellectual disability, language disorders, autism spectrum disorder, and other behavioral and emotional disorders.

NIDHI BEDI drnidhibedi@gmail.com 